\title{
3D Structure Studies of the Pancreatic Beta Cell by High Resolution Electron Microscope (EM) Tomography
}

\author{
B. J. Marsh*,**,***, A. J. Costin*, G. P. Morgan* and P. van der Heide* \\ *Institute for Molecular Bioscience, Queensland Bioscience Precinct, The University of Queensland, \\ Brisbane, QLD 4072, AUSTRALIA \\ **Centre for Microscopy and Microanalysis, The University of Queensland, Brisbane, QLD 4072, \\ AUSTRALIA \\ ***School of Molecular and Microbial Sciences, The University of Queensland, Brisbane, QLD \\ 4072, AUSTRALIA
}

The beta cells of the endocrine pancreas are the sole source of insulin in humans. Death of the beta cells, or their failure to make/release adequate amounts of insulin, results in the disease known as Diabetes. We are using large volume, dual-axis EM tomography to study the mechanisms involved in insulin biosynthesis, processing and trafficking in 3D at approximately $5 \mathrm{~nm}$ resolution in beta cells preserved in situ in intact pancreatic "islets of Langerhans" isolated from mice and humans. 300-400 nm-thick sections cut from high-pressure frozen, freeze-substituted and plastic-embedded beta cells/islets are imaged in a 300keV Technai F30 intermediate voltage EM (FEI), using motorized rotation holders capable of high tilt (GATAN). "Tilt series" image data are acquired automatically to a large format (4k), ultra-high sensitivity phosphor CCD camera (GATAN) as the sections are serially tilted through $0.75^{\circ}$ or $1^{\circ}$ increments over a range of $130-140^{\circ}$, about two orthogonal axes using the microscope control program SerialEM [1]. Semi-automated methods for CCD data acquisition, image montaging and image alignment by cross-correlation controlled by SerialEM help to minimize electron dose to the specimen. Tilt series images are brought into register with one another by cross-correlation, and then more accurately aligned by tracking the positions of small (5 or $10 \mathrm{~nm})$ gold fiducial markers on the surface(s) of the sections using a modification of the TILTALIGN program that uses subsets of fiducials to solve more accurately for local distortions/alignments $[2,3]$. Tomograms calculated by R-weighted back-projection from each set of aligned tilts are then matched to each other in 3D and combined to produce a single, high resolution 3D reconstruction [4]. 3D cellular reconstructions generated in this way and analyzed using the IMOD software package [5] have demonstrated the complexity of structural relationships among organelles of the insulin biosynthetic pathway $[3,6]$ and revealed novel connections between compartments that are normally spatially/functionally distinct [7]. To complement these insights into insulin trafficking in the beta cell to whole cell structure-function relationships, we have now undertaken to reconstruct an entire beta cell in $3 \mathrm{D}$ at $\leq 5 \mathrm{~nm}$ resolution. This reconstruction, which will be approximately 100-fold larger than any produced so far at comparable resolution [Marsh et al. (2001) PNAS 98:2399-2406], will by necessity require the parallel development of complex 3D algorithms for extracting useful structural/biological information from the data in a semi-automated manner [7,8]. The use of automated data mining tools, combined with the development of robotics instrumentation for loading/unloading specimens into the microscopes together with automated EM data collection routines [9-11], will mean that subsequent reconstructions of whole cells will be carried out exponentially faster in the future. Such a "Visible Cell" atlas will provide a unique structural framework which will serve as a major informatics/3D visualization/educational resource for the molecular cell biology, Diabetes and computational simulation communities. 
References

[1] D. N. Mastronarde, Microsc. Microanal. 9 (2003) 1182.

[2] D. N. Mastronarde et al., Microsc. Microanal. 7 (2001) 90.

[3] B. J. Marsh et al., Proc. Natl. Acad. Sci. USA. 98 (2001) 2399.

[4] D. N. Mastronarde, J. Struct Biol. 120 (1997) 343.

[5] J. R. Kremer et al., J. Struct. Biol. 116 (1996) 71.

[6] B. J. Marsh et al., Biochem. Soc. Trans. 29 (2001) 461.

[7] B. J. Marsh et al., Proc. Natl. Acad. Sci. USA. 101 (2004) 5565.

[8] N. Volkmann, J. Struct. Biol. 138 (2002) 123.

[9] C. S. Potter et al., Ultramicroscopy 77 (1999) 153.

[10] C. S. Potter et al., J. Struct. Biol. 146 (2004) 431.

[11] B. Carragher et al., J. Struct. Biol. 132 (2000) 33.

[12] BJM is supported by a Career Development Award from the Juvenile Diabetes Research

Foundation International (2-2004-275). The Boulder Laboratory for 3-D Electron Microscopy of Cells is supported by a National Institutes of Health (NIH) National Center for Research Resources (NCRR) Biomedical Technology Grant (P41 RR00592) to J. Richard McIntosh. Much of the work discussed here was additionally supported by funding from the National Institutes of Health to Kathryn E. Howell (P01-GM61306). 